\title{
A Two-Stage Multi-Objective Enhancement for Fused Magnetic Resonance Image and Computed Tomography Brain Images
}

\author{
Leena Chandrashekar* \\ Research Scholar, R V College of Engineering, Visvesvaraya Technological University, India \\ Leenamaheshnikam10@gmail.com \\ Sreedevi A \\ Associate Professor, R V College of Engineering, Visvesvaraya Technological University, India \\ sreedevia@rvce.edu.in
}

Received: 04/Oct/2019 Revised: 24/Jan/2020 Accepted: 08/Feb/2020

\begin{abstract}
Magnetic Resonance Imaging (MRI) and Computed Tomography (CT) are the imaging techniques for detection of Glioblastoma. However, a single imaging modality is never adequate to validate the presence of the tumor. Moreover, each of the imaging techniques represents a different characteristic of the brain. Therefore, experts have to analyze each of the images independently. This requires more expertise by doctors and delays the detection and diagnosis time. Multimodal Image Fusion is a process of generating image of high visual quality, by fusing different images. However, it introduces blocking effect, noise and artifacts in the fused image. Most of the enhancement techniques deal with contrast enhancement, however enhancing the image quality in terms of edges, entropy, peak signal to noise ratio is also significant. Contrast Limited Adaptive Histogram Equalization (CLAHE) is a widely used enhancement technique. The major drawback of the technique is that it only enhances the pixel intensities and also requires selection of operational parameters like clip limit, block size and distribution function. Particle Swarm Optimization (PSO) is an optimization technique used to choose the CLAHE parameters, based on a multi objective fitness function representing entropy and edge information of the image. The proposed technique provides improvement in visual quality of the Laplacian Pyramid fused MRI and CT images.
\end{abstract}

Keywords - Glioblastoma; Laplacian Pyramid; Image Fusion; Image Enhancement; Contrast Limited Adaptive Histogram Equalization; Particle Swarm Optimization

\section{1- Introduction}

Glioblastoma is the fastest growing Grade IV malignant tumors found in the brain with a survival time of less than a year after their detection [1]. The detection of these tumors has always been a challenge to doctors. Some of the reasons for delay in detection are due to failure to understand the early symptoms, lack of awareness, inadequate healthcare facilities like imaging, preliminary screening for the patients and expertise with doctors. An inability to distinguish tumors by doctors and experts is mostly due to incorrect imaging procedures, patient's condition at the time of image acquisition and noise may lead to delayed prognosis. For this reason, medical imaging is paramount in detection, identification, grading and diagnosis of the Glioblastoma. Doctors recommend many imaging techniques for detection of Glioblastoma like Computed Tomography (CT), Magnetic Resonance Imaging (MRI) and its variants, Fluid Attenuated Inverse Recovery (FLAIR) and Positron Emission Tomography (PET) [2]. These images are acquired sequentially through different scanning machines at different times. Each of the modalities provide different information of the brain. For example, the CT image provides the structural information of the brain like bone structure, tissue symmetries, changes in tissue density and space occupying lesions [3]. It also shows changes made in the nearby skull region due to tumor extension and calcification of tumors. Conversely, CT images fail to indicate tumor borders and infiltration in the nearby regions. These can be easily visualized with MR (Magnetic Resonance) images, which provide structural and functional information of the brain along with high contrast and resolution for soft tissues like tumors or lesions [3].

FLAIR images are a special type of MRI, which is sensitive to changes at the periphery of the cerebral hemispheres. PET images assess the tumor growth and spread [4]. These multimodal images are the noninvasive ways to detect Glioblastoma. Based on the multimodal images, surgical resection or complete removal of tumor is made followed by radio chemotherapy [4]. Thus, a single imaging technique is never sufficient to confirm the presence or extent of the tumor [5]. The latest development in medical imaging is generation of a complete image, obtained by fusing the multimodal images to a single 
image [6]. This helps in early detection of tumors and requires lesser digital storage [7-8]. Some of the techniques for fusing the multimodal images are Discrete Wavelet Transform (DWT), Laplace Transform (LT), Contourlet Transform (CT) and Non-sub Sampled Contourlet transform (NSCT) [9]. The process of fusion begins with decomposing the multimodal images in to approximation coefficients (low frequency components of the image) and detailed coefficients (high frequency components) using the above techniques. These components are combined using different rules like averaging, summing, weighted summing, max-min or max-max fusion rules [10]. The fused coefficients are recomposed using inverse transforms to generate a fused image. Fig. 1 shows the methodology of the fusion process using DWT [10]

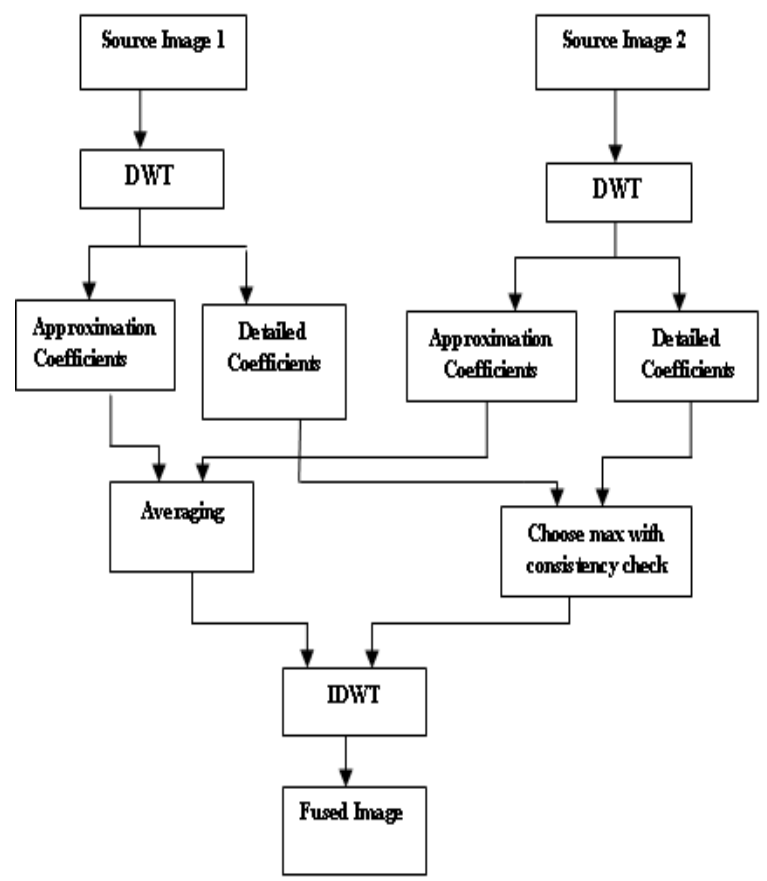

Fig.1.Methodology for Multimodal Image Fusion

The visual quality of the fused image is evaluated based on contrast, edge information and peak signal to noise ratio. Contrast is measured in terms of standard deviation of the image and is significant in fused images, because it helps to distinguish the healthy and tumorous cells. The edge information in the image represents the tumor boundary and also assists in locating the tumor. Noise exists at the time of image acquisition due to various reasons like patient position, scanner machines or in processing the MRI or CT machine. Peak signal to noise ratio indicates the signal to noise ratio in the fused image. The fusion process introduces blocking effect, noise and artifacts that greatly reduce the visual quality of the fused image. Fig. 2 shows a fused CT and MR image obtained by DWT indicating blocking effect at the edges and corners [10]. The fused images with poor visual quality make it difficult for experts to interpret the tumor presence or its spread. Hence, there is a need for an enhancement technique to improve the visual quality and reduce blocking effect, noise and artifacts. Most of the enhancement techniques deal with improving image contrast, as it helps in differentiating the Glioblastoma and the normal cells. Nevertheless, the drawback with these techniques is that it only increases the dynamic range of the image, which is a function of pixel intensity alone. Tumors or any abnormality in the brain appears distinct, bright or light intensity in MR images. Thus, by varying the contrast, the normal and the tumorous cells can be differentiated easily. On the contrary, the high contrast images or low contrast MR image makes it challenging to differentiate the Glioblastoma [6]. The contrast enhancement is mostly a 2fold process, consisting of contrast stretch and tonal enhancement. The contrast stretch improves the brightness differences uniformly across the dynamic range of the image and tonal enhancement improve the brightness differences in different areas like dark, gray or bright regions in the image [6]. The paper deals with enhancement techniques for fused images - contrast enhancement, improvement in entropy and structural information.

\section{2- Enhancement Techniques}

Generally, the contrast enhancement is achieved by the following techniques - Non-Linear Transfer function, Histogram based and Frequency Domain [11]. Among them, Histogram Equalization (HE) is the most popular
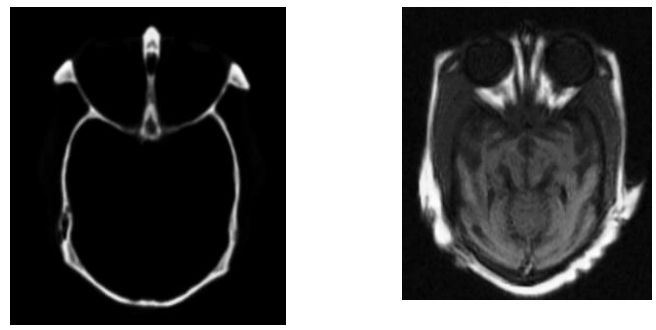

(a) (b)

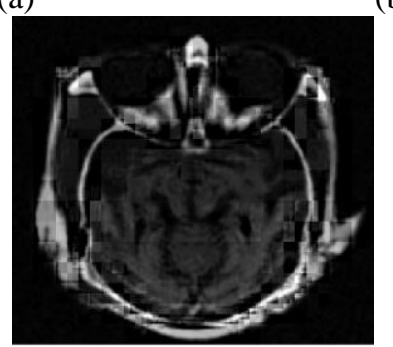

(c)

Fig.2 (a) CT image (b) MR image (c) Fused image 
technique for contrast enhancement performed in a spatial domain. This deals with remapping the gray scale values of the original input image to a new level of gray scale values. The transformation is made by a simple scaling of values or by use of linear or non-linear functions. Consequently, HE attains the contrast enhancement by flattening and stretching the dynamic range of the image's histogram. The enhanced image shows improved characteristics like contrast, entropy, peak signal to noise ratio and edge information with respect to the original image. Although $\mathrm{HE}$ is a popular enhancement technique, it suffers from visual artifacts like intensity saturation and amplification due to large number of homogenous pixels. Moreover, the equalization is accomplished uniformly for all the pixels of the image, leading to enhanced global contrast. However, the lowest intensity pixels become less significant, thereby reducing the local contrast [12]. In order to overcome the drawbacks of HE, newer enhancement techniques are proposed for improved visual quality with the use of median filters, adaptive gamma correction and homomorphic filtering [13-14].

An Adaptive Histogram Equalization (AHE) technique is a block based adaptive method, which is capable of reducing the drawbacks of HE. It deals with the local contrast rather than global contrast. The local contrast is more significant in the detection of Glioblastoma. In this technique, histogram equalization is performed on sub-images or small and equal sized blocks obtained by splitting the image. The equalization is executed on every block independently and mapped to new intensity levels based on a transformation function. The new pixel values are solely based on the neighboring pixel characteristics. Then, bilinear interpolation is used to combine the blocks after equalization [14]. The major challenge with AHE is the selection of the block size and the transformation function, which are significant and greatly affect the quality of the enhanced image. However, AHE suffers from blocking effect, at the time of combining the blocks. In addition to blocking effect, over-amplification is also seen due to large homogenous regions of the image. Youlian Zhu et al have proposed an adaptive histogram equalization technique for CT images. A user defined parameter $\beta$, is suggested based on the gray level of the image. The entropy is used as an objective function to select the $\beta$ adaptively [15].

A variant of AHE is the Contrast Limited Adaptive Histogram Equalization (CLAHE), proposed by $\mathrm{K}$ Zuierveld, is also a block-based contrast enhancement technique with focus on local contrast. Unlike AHE, CLAHE provides uniform equalization with clipping the excess portion of large peaks found after the histogram equalization, thereby avoiding over-amplification. The excess portion removed depends on a parameter called clip limit, which is a function of the dynamic range of the image and block size. CLAHE involves setting of three operational parameters - clip limit, block size and distribution function, which must be selected before performing the image enhancement to achieve good contrast images, free from noise and artifacts [16]. Various histogram based enhancement techniques are compared and analyzed, CLAHE is observed to perform better for MRI brain Images [17].

The simplest technique of setting the operational parameters for CLAHE is by trial and error. However, this is time consuming, may deviate from the actual values and changes for every image. Some of the techniques used to set the parameters are based on textureness of the image, maximum curvature of entropy, Least Mean Square (LMS) algorithm, multi-objective optimization technique and fuzzy rules. Despite various techniques for contrast enhancement, CLAHE seems to provide good local contrast, however it largely fails to enhance the pixels with low gray level intensity. Moreover, there is no standard for finding the optimal clip limit for a specific region of interest in medical images. Generally, clip limit is proportional to the multiple of average height of the histogram, where the multiplication factor is user-defined and varies for different images, which is the major drawback of CLAHE. Therefore, there is a need to choose clip limit adaptively for every block of image without any user intervention [23].

The clip limit is also a function of dynamic range of the grayscale image, block size and slope of transformation function. Initially, the clip limit and block size is chosen empirically and then obtain the optimal values based on statistical parameters like entropy, peak signal to noise ratio or edge information. Yet clip limit may change depending on the type of images. This makes the enhancement process very extensive and time consuming [23]. Moreover, inaccurate selection of clip limit can cause over-amplification in CLAHE. Bilateral Filter and Median filters are used to overcome this drawback [24-25].

Optimization techniques provide a convenient way of determining the CLAHE parameters without any heuristics and compute them adaptively for every image block. Particle Swarm Optimization (PSO) - a population-based optimization technique proposed by Eberhart and Kennedy [27]. The motivation for PSO is from the biological social groupings of animals, which interact with each other to find food or save each other from predators. It uses swarm intelligence to solve any optimization problem. A swarm is a group of possible solutions also called particles, which provide a solution to the optimization problem. The performance of the optimization is evaluated based on a fitness function. Each particle of the swarm is identified with its velocity and position, which are updated through 
iterations. The search for best solution terminates at the end of the iterations or when the solution generates the highest fitness value [27]. Malik Braik and Alaa Sheta have implemented the PSO algorithm for enhancing general images [28].

The objective of this paper is to enhance the contrast of Multimodal and Multiresolution fused images, obtained by a fusing MRI and CT brain images. The fusion process induces blocking effect, noise and artifacts that greatly reduce the visual quality of the fused image. CLAHE is used to improve the contrast of the fused image. The structural information is of great importance in the fused MRI and CT images, as they indicate the periphery of tumor region. The PSO algorithm is used to select of the operational parameters of CLAHE automatically as well as enhance the image quality based on entropy and edge information. The operational parameters for them are set manually or empirically, however they change with images, making it tedious to set them manually every time.

\section{3- Enhancement Technique for Fused MRI and CT Image}

The research work includes preprocessing of MRI and CT images, image registration, and image fusion followed by image enhancement. More than 200 MRI and CT images containing Grade IV tumors - Glioblastoma is taken from www.Radiopedia.org for this work. There are quite a few databases available publicly for MRI images, but the challenge in our research is to get multimodal images for the same patient. Since these are acquired at different times and from different machines, they must be registered and preprocessed before fusing them. The Fig.3 shows the block diagram for the enhancement process. The preprocessing stage deals with resizing the image to size $256 \times 256$ and converting them to gray scale. The images contain Gaussian and Rician noises, which need to be eliminated; Non-Local Means Filter is used in filtering the $\mathrm{CT}$ and MRI images [29].

The preprocessing is followed by image registration mandatory step, where both the images are matched for size, orientation and scaling. Subsequently, the images are fused using Laplacian Pyramid [31], since it provides excellent contrast for fused images. The fusion process introduces blocking effect and noise in the fused image, thereby reducing the image quality as discussed in section 1 [30]. Consequently, the enhancement technique is required to enhance the fused image with minimum loss of original information. Since the focus is the tumor region, adaptive block-based enhancement technique like CLAHE is chosen.

The operational parameters for CLAHE are block size, clip limit and distribution function. The proposed technique is executed for different block sizes like $2 \times 2,3 \times 3,4 \times 4,5 \times 5$, $7 \times 7,8 \times 8$ and $10 \times 10$. The $8 \times 8$ provided superior results in terms of contrast and structural information, hence chosen to be constant for the enhancement process. There is a trade-off for high contrast large block size is considered and for high edge information low block size is chosen. Similarly, the enhancement process was performed with the various distribution functions like Uniform, Rayleigh and Exponential distribution. The Uniform distribution performed better than the other distribution functions. Hence, uniform distribution is considered in the proposed enhancement process. The clip limit is initialized randomly in the range of 0 to 0.01 , the PSO algorithm adaptively chooses the clip limit based on a fitness function. The proposed enhancement process is two-stage; firstly, the contrast of the MRI and CT fused image is enhanced by CLAHE algorithm, which increases the dynamic range of the image. Secondly, the multi-objective fitness function assists in choosing the clip limit that maximizes the entropy and edge information of the image. The Particle Swarm Optimization (PSO) algorithm is used to find the optimal clip limit. It begins with initialization of a group of 50 particles called as a swarm. In this work, the particles in the swarm represent the values for clip limit randomly chosen in the range of 0 to 0.01 . The fitness function given by Eq. (1) helps to determine the best clip limit.

$$
\mathrm{F}\left(\mathrm{I}_{\mathrm{e}}\right)=\log \left(\log \left(\mathrm{E}\left(\mathrm{I}_{\mathrm{s}}\right)\right)\right) \times \frac{\text { n_edges }\left(\mathrm{I}_{\mathrm{s}}\right)}{\mathrm{M} \times \mathrm{N}} \times \mathrm{H}\left(\mathrm{I}_{\mathrm{s}}\right)
$$

The cost function/fitness function is a product of entropy, sum of edge intensities and number of edge pixels. Since, multiple parameters are considered to measure the degree of enhancement; this function is called multi-objective function. Every particle $i$ in the swarm is represented by two parameters velocity and position. For any particle 'i', the position and velocity indicate its location in the swarm and fitness value respectively. These two parameters are initialized with some random values and is updated in every iteration using Eq. (2) and Eq. (3).

$$
\begin{aligned}
v_{i}(t+1)= & w v_{i}(t)+c_{1} r_{1}\left(p_{i}(t)-x_{i}(t)\right)+ \\
& c_{2} r_{2}\left(g(t)-x_{i}(t)\right) \\
x(t+1)= & x_{i}(t)+v_{i}(t+1)
\end{aligned}
$$

where $\mathrm{v}_{\mathrm{i}}(\mathrm{t})$ and $\mathrm{x}_{\mathrm{i}}(\mathrm{t})$ represent the velocity and position for an particle $\mathrm{i}$ and iteration t. Eq.(2) comprises of three components - first component representing the initial velocity of the particle, the second component represents the particle's decision based on its own experience and the third component indicates the particle's decision based on swarm's experience. In every iteration, the image is enhanced using CLAHE with the 


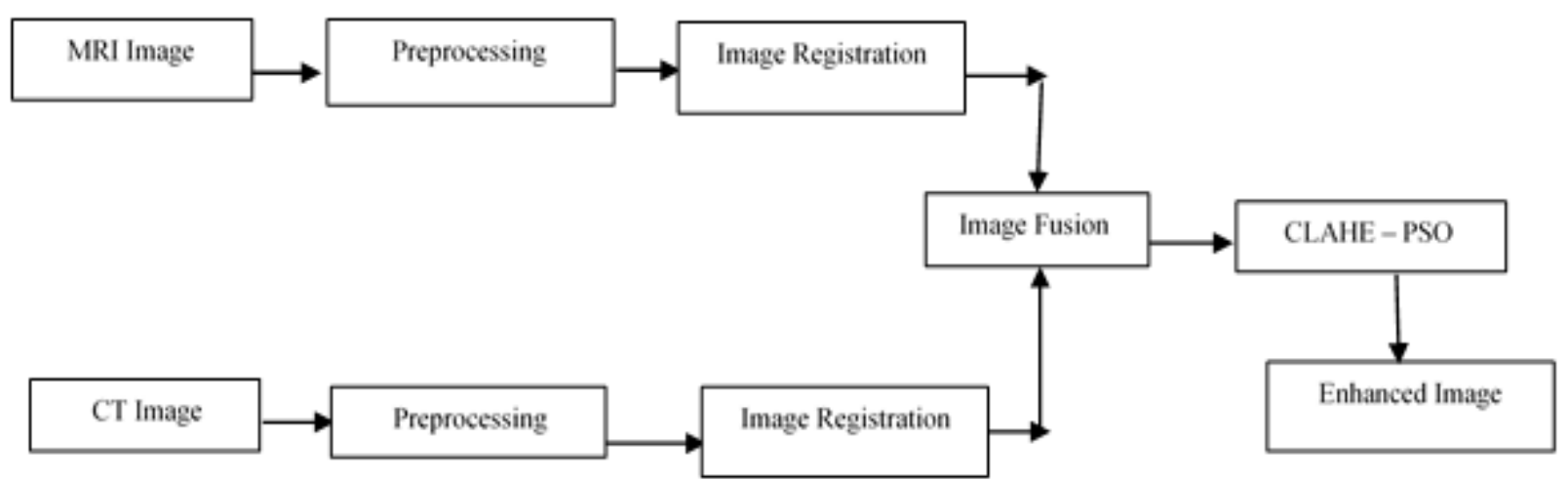

Fig.3 Block diagram for the Enhancement Process

selected clip limit (each particle). The fitness function is computed using Eq. (1). This process is repeated for all the particles to get the best fit (clip limit). The clip limit that maximizes the fitness function can be accessed from the swarm based on its position and velocity and is represented as 'pbest' or $\mathbf{p}_{\mathbf{i}}(\mathbf{t})$. This denotes the best local solution for that iteration. The enhancement process is repeated for all the iterations to get 'pbest' or $p_{i}(t)$ for each iteration. In case the 'pbest' value in the current iteration is greater than the previous one, then the 'pbest' is updated with a new 'pbest' and 'gbest', otherwise the 'pbest' from previous iteration is retained as 'pbest' and 'gbest'. The 'gbest' or $\mathrm{g}(\mathrm{t})$ in Eq.(2) is the global solution for the enhancement process obtained at the end of all the iterations. When 'pbest' appears equal 'gbest' over a predefined number of iterations the enhancement process terminates.

Initialize the particle swarm

For each iteration

For each particle

Enhance the image using CLAHE

Compute the fitness value for the enhanced image as per Eq. (1) for every particle

If the fitness value is greater than the previous fitness value (pbest)

$$
\text { End }
$$

Set current value as the new pbest (gbest)

Choose the particle with the best fitness value among all the pbest (gbest)

For each particle

Calculate particle velocity as per Eq. (2)

Calculate the particle position as per Eq. (3)
End

Continue while maximum iterations are attained.

End

Report the gbest and pbest

Fig.4 Pseudo code for Proposed Technique (CPSO)

A balance between 'pbest' and 'gbest' is achieved by inertia weight represented as $\mathrm{w}, \mathrm{c} 1$ and $\mathrm{c} 2$ - the positive acceleration constants and $\mathrm{r} 1$ and $\mathrm{r} 2$ are random values in the range of $[0,1]$. The, an optimal value for the clip limit is obtained by PSO algorithm based on maximum value of the fitness function [32]. Fig. 2 shows the pseudo code for proposed CLAHE-Particle Swarm Optimization (CPSO) algorithm.

\section{4- Experiment and Results}

The CT and MRI images prior to enhancement are preprocessed, registered and fused using Laplacian Pyramid [31]. The dataset includes many variants for CT and MRI images - (i) images with contrast agent (ii) images without contrast agent and (iii) delayed images with contrast agent. The combination of CT and MRI T1 image, CT and MRI T2 and CT and MRI FLAIR images are used to generate the fused image. The proposed enhancement technique is tested on more than 200 MRI and CT fused images, containing the different types of Glioblastoma - Multicentric Glioblastoma, Multifocal Glioblastoma, Cystic Glioblastoma and Giant Cell Glioblastoma. 


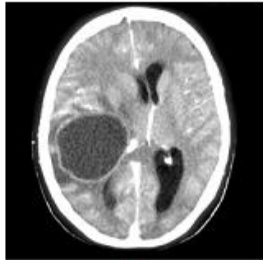

(a)

Fig. 5 Dataset D20

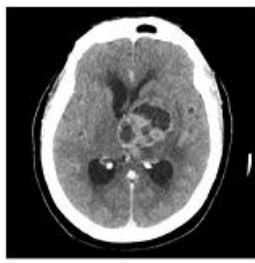

(a)

Fig. 6 Dataset D53

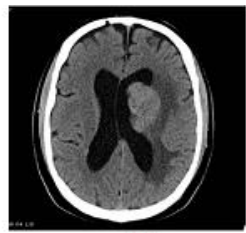

(a)

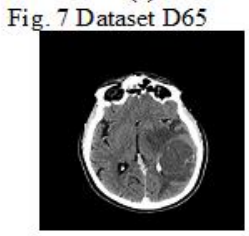

(a)

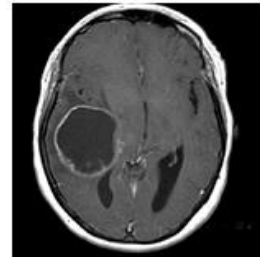

(b)

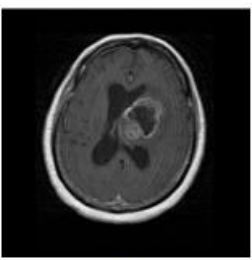

(b)

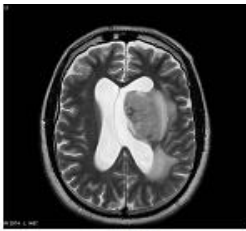

(b)

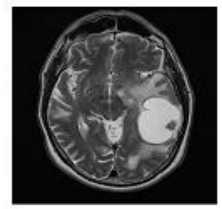

(b)

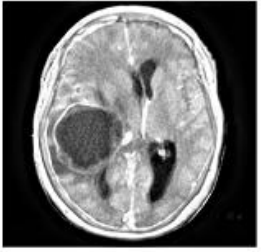

(c)

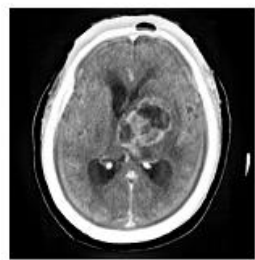

(c)

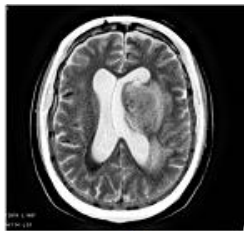

(c)

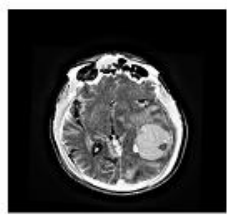

(c)

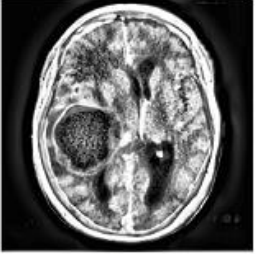

(d)

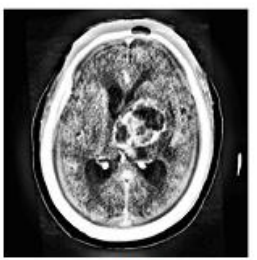

(d)

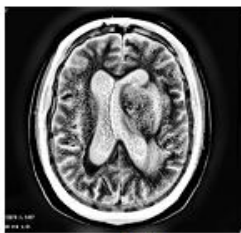

(d)

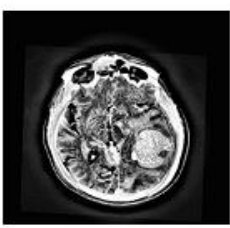

(d)

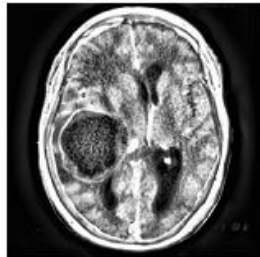

(e)

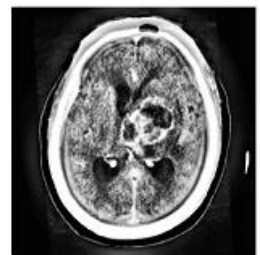

(e)

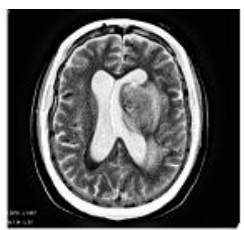

(e)

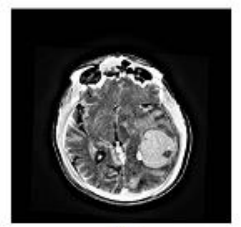

(e)

Fig. 8 Dataset D65

Table 1: Characteristics for Dataset D20 - D50

\begin{tabular}{|c|c|c|c|c|c|}
\hline Dataset & CT & MRI & Size of Tumor & Group & Type of tumor \\
\hline D20 & High Contrast & Low contrast & Large & I & Cystic High Grade Glioblastoma \\
\hline D53 & Large size & Small size & Large & I & Glioblastoma (Grade IV) \\
\hline D60 & Large Size & Large Size & Large & I & Giant cell Glioblastoma \\
\hline D67 & Large size & Small size & Large & I & Primary CNS Lymphoma \\
\hline D65 & Small size & $\begin{array}{c}\text { Large size, Poor } \\
\text { contrast }\end{array}$ & Large & I & $\begin{array}{c}\text { differential diagnosis - metastasis } \\
\text { and glioblastoma }\end{array}$ \\
\hline D17 & Misaligned & $\begin{array}{c}\text { Aligned, Poor } \\
\text { contrast }\end{array}$ & Small & II & Glioblastoma (Grade IV) \\
\hline D16 & Aligned & $\begin{array}{c}\text { Misaligned, Poor } \\
\text { contrast }\end{array}$ & Small & II & Multicentric Glioblastoma \\
\hline D15 & Aligned & $\begin{array}{c}\text { Misaligned, Poor } \\
\text { contrast }\end{array}$ & Small & II & Multifocal glioblastoma \\
\hline D6 & Large & Small, Poor contrast & Small & II & Glioblastoma (Grade IV) \\
\hline D50 & Small & Large & Small & II & Glioblastoma (Grade IV) \\
\hline
\end{tabular}




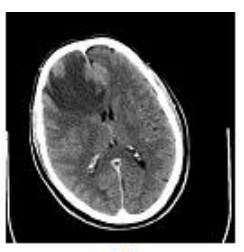

(a)

Fig. 9 Dataset D17

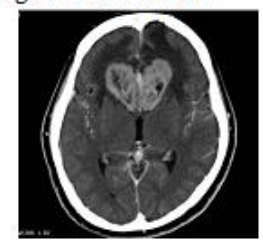

(a)

Fig. 10 Dataset D60

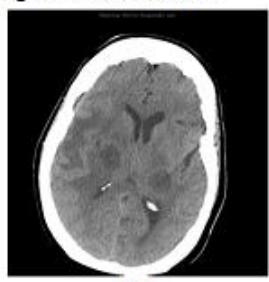

(a)

Fig. 11 Dataset D16

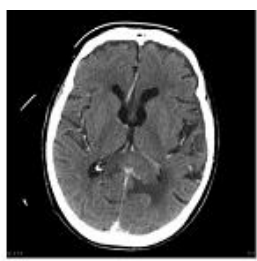

(a)

Fig. 12 Dataset D 15

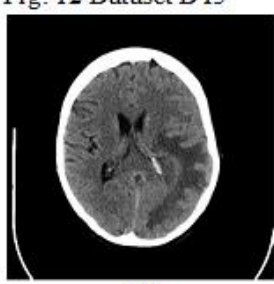

(a)

Fig. 13 Dataset D6

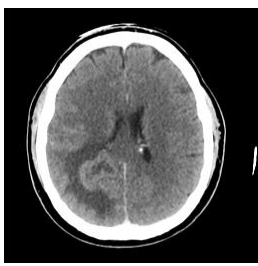

(a)

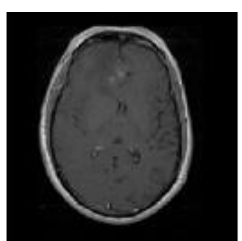

(b)

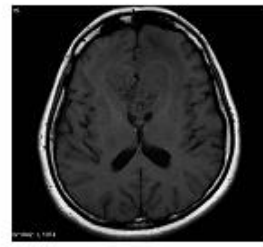

(b)

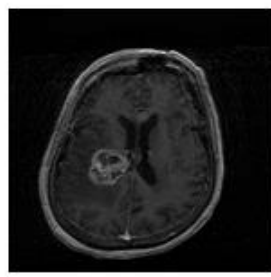

(b)

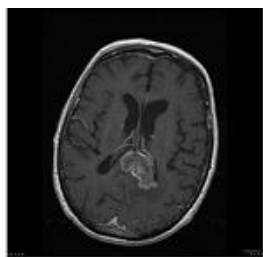

(b)

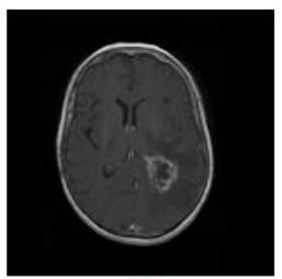

(b)

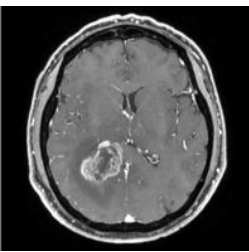

(b)

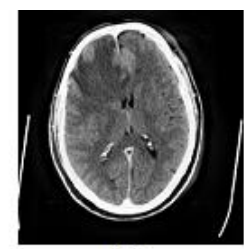

(c)

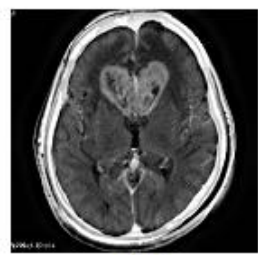

(c)

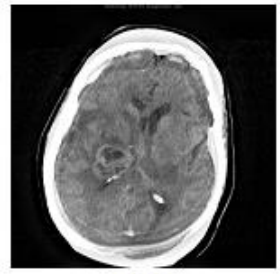

(c)

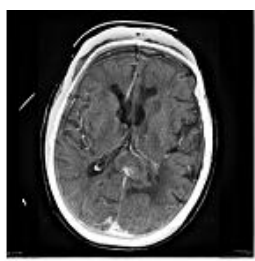

(c)

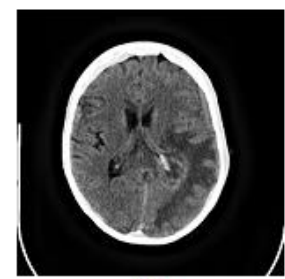

(c)

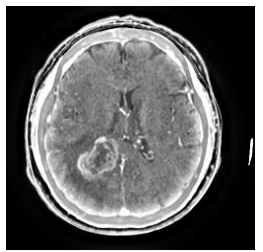

(c)

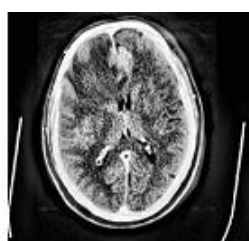

(d)

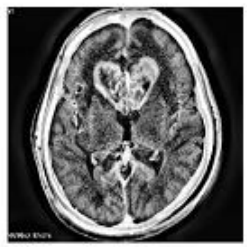

(d)

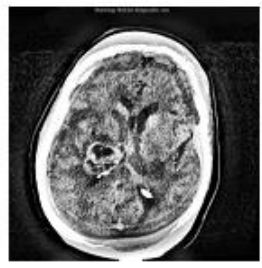

(d)

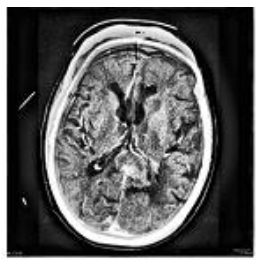

(d)

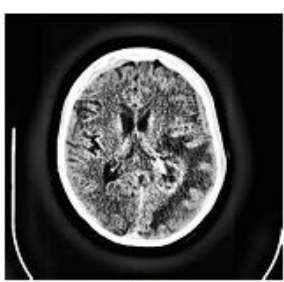

(d)

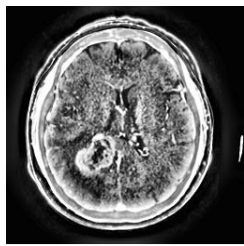

(d)

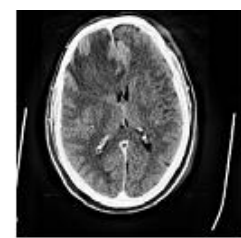

(e)

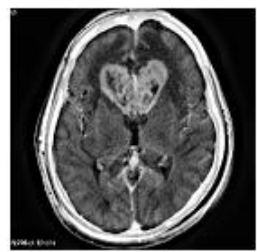

(e)

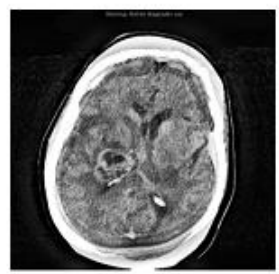

(e)

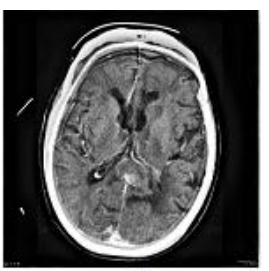

(e)

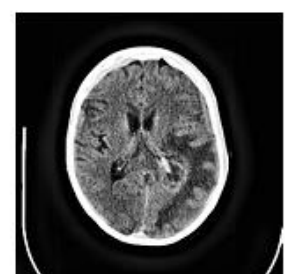

(e)

Fig. 14 Dataset D50

Fig.5-14 Dataset D20 - D50 (a) CT Image (b) MRI Image (c) Fused Image (d) CLAHE (e) Proposed 
Table 2: The Performance Parameters for Evaluation of the Enhanced image

\begin{tabular}{|c|c|c|}
\hline S.no & Parameter & Equation \\
\hline 1. & Standard Deviation (SD) & $\sqrt{\frac{1}{\mathrm{HxW}} \sum_{\mathrm{x}=1 \mathrm{y}=1}^{\mathrm{H}} \sum^{\mathrm{W}}(\mathrm{F}(\mathrm{x}, \mathrm{y})-\mu)^{2}}$ \\
\hline 2. & Entropy (En) & $\sum_{\mathrm{l}=0}^{\mathrm{L}-1} \mathrm{p}(\mathrm{l}) \log _{2} \mathrm{p}(\mathrm{l})$ \\
\hline 3. & Mean Square Error (MSE) & $\frac{1}{\mathrm{MN}} \sum_{\mathrm{i}=1}^{\mathrm{N}} \sum_{\mathrm{j}=1}^{\mathrm{N}}\left(\mathrm{I}_{1}(\mathrm{i}, \mathrm{j})-\mathrm{E}(\mathrm{i}, \mathrm{j})\right)^{2}$ \\
\hline 4. & Peak Signal to Noise Ratio (PSNR) & $20 \log _{10}\left(\frac{255}{\sqrt{\mathrm{MSE}}}\right)$ \\
\hline 5. & Structural Similarity Index Metric (SSIM) & $\frac{\left(2 \mu_{e} \mu_{\mathrm{s}}+\mathrm{C}_{1}\right)\left(2 \sigma_{e}+\mathrm{C}_{2}\right)}{\left(\mu_{\mathrm{e}}^{2}+\mu_{\mathrm{s}}^{2}+\mathrm{C}_{1}\right)\left(\sigma_{\mathrm{e}}^{2}+\sigma_{\mathrm{s}}^{2}+\mathrm{C}_{2}\right)}$ \\
\hline 6. & Universal Image Quality Index (UIQI) & $\frac{4 \sigma_{e} \sigma_{\mathrm{s}}\left(\mu_{\mathrm{e}}+\mu_{\mathrm{s}}\right)}{\left(\sigma_{\mathrm{e}}^{2}+\sigma_{\mathrm{s}}^{2}\right)\left(\mu_{\mathrm{e}}^{2}+\mu_{\mathrm{s}}^{2}\right)}$ \\
\hline
\end{tabular}

Table 3: Experimental Results for Dataset D20 - D50

\begin{tabular}{|c|c|c|c|c|c|c|c|c|c|}
\hline \multicolumn{4}{|c|}{ STD } & \multicolumn{3}{|c|}{ En } & \multicolumn{3}{|c|}{ SSIM } \\
\hline Dataset & Fused & CLAHE & Proposed & Fused & CLAHE & Proposed & Fused & CLAHE & Proposed \\
\hline D20 & 91.32 & 85.24 & 85.41 & 6.51 & 6.81 & 6.78 & 0.5 & 0.70 & 0.73 \\
\hline D53 & 84.77 & 86.03 & 85.77 & 6.24 & 6.65 & 6.49 & 0.74 & 0.65 & 0.82 \\
\hline D67 & 70.13 & 78.01 & 71.84 & 6.57 & 7.15 & 6.75 & 0.75 & 0.65 & 0.92 \\
\hline D65 & 83.34 & 85.03 & 83.79 & 6.32 & 6.79 & 6.32 & 0.64 & 0.69 & 0.96 \\
\hline D60 & 72.81 & 75.03 & 73.02 & 4.92 & 5.30 & 4.96 & 0.73 & 0.59 & 0.93 \\
\hline D17 & 79.54 & 80.34 & 80.25 & 6.23 & 6.79 & 6.78 & 0.55 & 0.62 & 0.64 \\
\hline D16 & 78.29 & 80.19 & 79.18 & 5.60 & 6.19 & 6.11 & 0.64 & 0.59 & 0.66 \\
\hline D15 & 84.24 & 81.39 & 81.85 & 6.28 & 7.02 & 6.69 & 0.55 & 0.60 & 0.80 \\
\hline D6 & 74.42 & 80.23 & 77.42 & 5.30 & 5.72 & 5.54 & 0.54 & 0.57 & 0.76 \\
\hline D50 & 73.59 & 80.09 & 75.71 & 6.22 & 6.60 & 6.34 & 0.56 & 0.64 & 0.92 \\
\hline \multicolumn{4}{|c|}{ UIQI } & \multicolumn{3}{|c|}{ PSNR } & \multicolumn{3}{|c|}{ MSE } \\
\hline Dataset & Fused & CLAHE & Proposed & Fused & CLAHE & Proposed & & AHE & Proposed \\
\hline D20 & 0.51 & 0.73 & 0.72 & 16.22 & 19.77 & 20.86 & & 35.3 & 532.63 \\
\hline D53 & 0.75 & 0.63 & 0.70 & 24.62 & 21.43 & 25.49 & & 7.07 & 183.57 \\
\hline D67 & 0.72 & 0.70 & 0.95 & 16.80 & 18.17 & 27.50 & & 9.00 & 115.60 \\
\hline D65 & 0.64 & 0.73 & 0.93 & 13.41 & 21.50 & 34.87 & & 9.57 & 21.16 \\
\hline D60 & 0.72 & 0.33 & 0.66 & 20.13 & 23.65 & 36.39 & & 0.46 & 14.92 \\
\hline D17 & 0.55 & 0.62 & 0.64 & 22.37 & 21.59 & 22.20 & & 0.82 & 391.61 \\
\hline D16 & 0.62 & 0.47 & 0.59 & 25.80 & 21.45 & 23.77 & & 5.56 & 272.78 \\
\hline D15 & 0.50 & 0.55 & 0.92 & 26.47 & 21.08 & 26.85 & & 6.09 & 134.15 \\
\hline D6 & 0.49 & 0.47 & 0.43 & 30.32 & 20.92 & 25.99 & & 7.74 & 163.66 \\
\hline D50 & 0.48 & 0.58 & 0.95 & 21.61 & 19.13 & 27.34 & & 3.91 & 119.88 \\
\hline
\end{tabular}




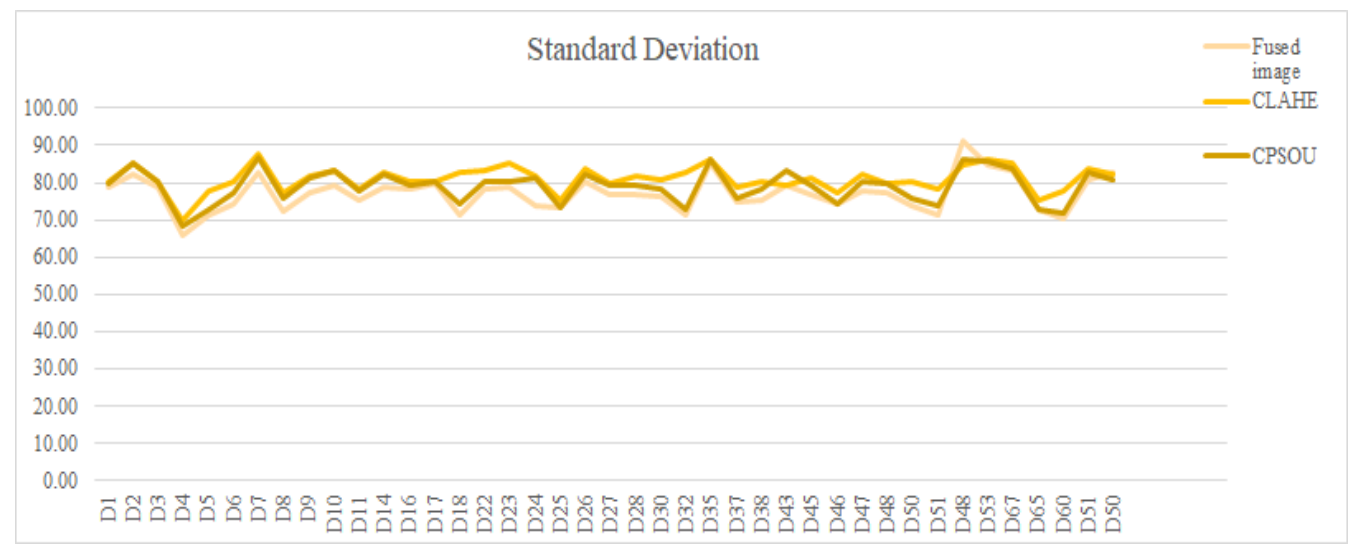

Fig.15 Standard Deviation

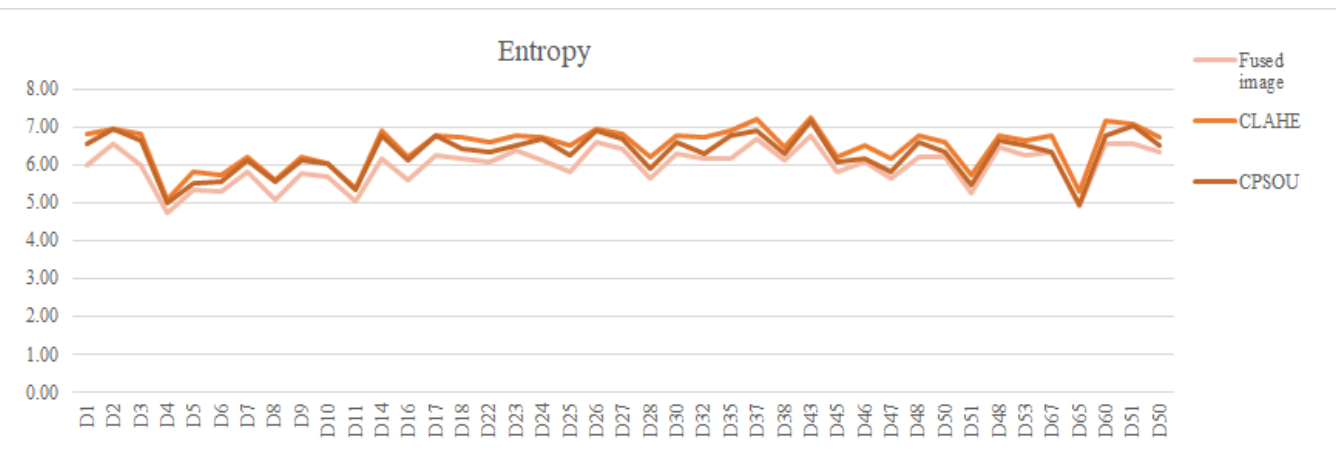

Fig.16 Entropy

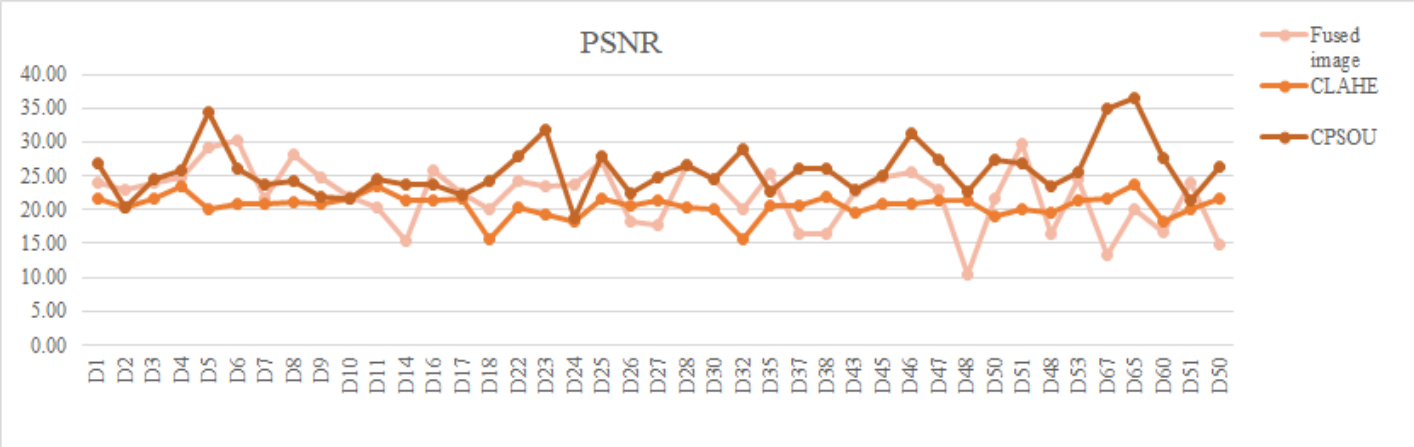

Fig. 17 PSNR

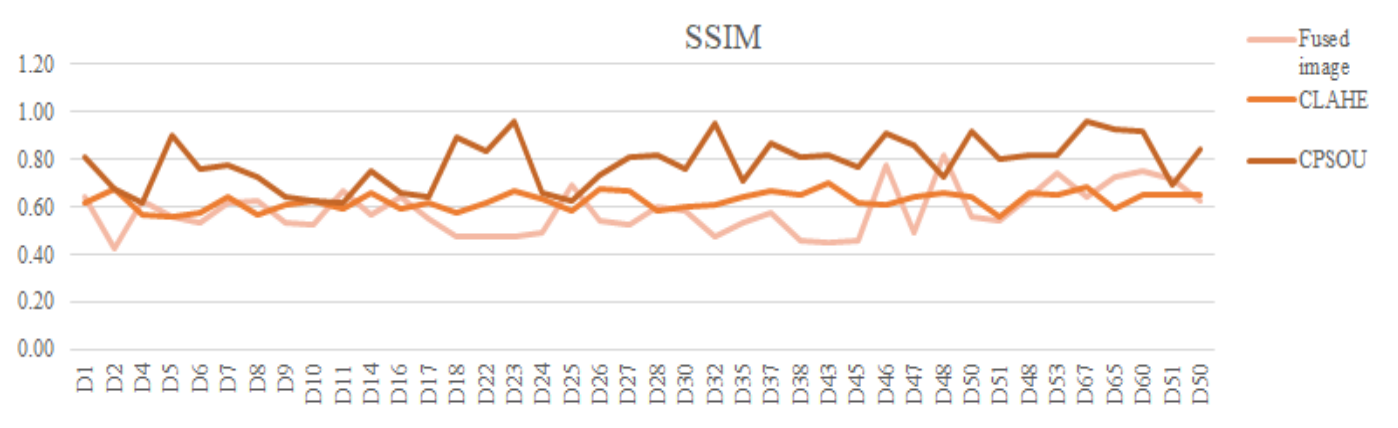

Fig.18 SSIM 
tissues and tumors. The information content of the fused image is

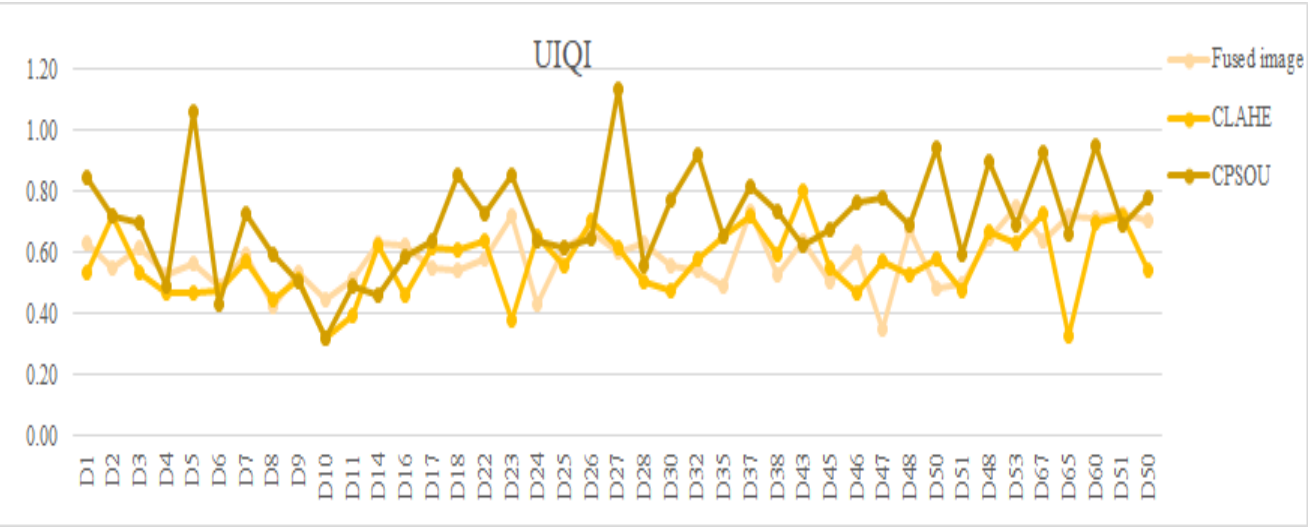

Fig.19 UIQI

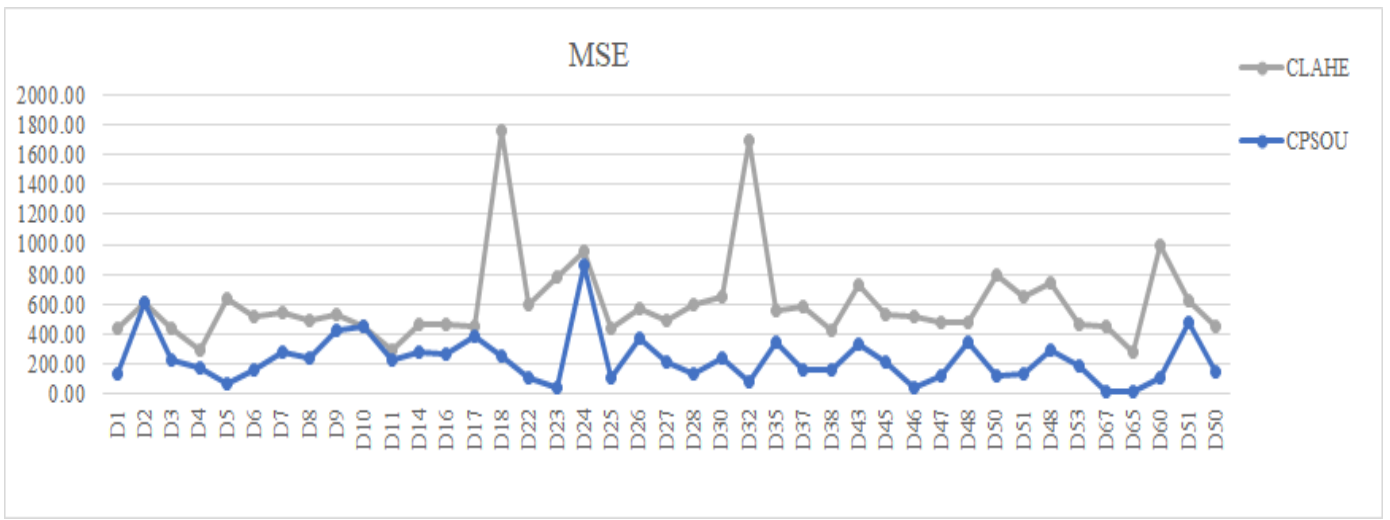

Fig.20 MSE

Fig. 5-14 shows some sample CT image, MRI image, fused image, CLAHE enhanced image and the CPSO (proposed) enhanced image for 10 datasets D20-50. Dataset belonging to Group I represent large tumors and Group II contain images with small tumors. In addition, high contrast CT and poor contrast MRI images are also tested with the proposed technique. For example, the CT image in dataset 20 has very high contrast and MRI T1 in dataset 16 and 17 has poor contrast. The proposed enhancement technique is implemened for fused CT and MRI image generated in [30-31], thus the performance parameters are the same. Standard Deviation (SD), Entropy (En), Structural Similarity Index (SSIM), Peak Signal to Noise Ratio (PSNR), Unique Image Quality Index (UIQI) and Mean Square Error (MSE) are considered to measure the quality of the enhanced image and compared with the fused image quality. The Table 2 shows the expressions for various performance parameters, the detailed definitions are in [30].

The Standard Deviation (SD) represents the contrast of the enhanced image. It helps in differentiating the soft measured by Entropy. The ratio of the maximum pixel intensity to Mean Square Error(MSE) in the enhanced image is indicated by Peak Signal To Noise Ratio (PSNR) MSE is a measure of similiarity between the orginial image and the enhanced image. Structural Similarity Index Metric (SSIM) is a measure of the structural similiarity between the source image and enhanced image. The value of SSIM lies in the range of 0 and 1 . The value 1 or close to 1 indicates high structural similiarity between the source image and enhanced image. Universal Image Quality Index (UIQI) represents coefficient correlation, illumination and contrast of enhanced image and source image. Fig.15-20 indicate the results for all that datasets and performance parameters. An efficient enhancement technique can provide high STD, En, PSNR, SSIM, UIQI and a minimum MSE. It can be observed that images enhanced by CLAHE seem enhanced, however it is only in terms of contrast or variaion of brightness but the structural information is poor. 
Although, the standard deviation and entropy is high for enhanced image obtained by CLAHE, the PSNR, SSIM, UIQI is lesser than those obtained with the proposed enhancement technique.This is because, the CLAHE deals with enhancing the dynamic range and increasing the high intensity pixels and further decreasing the low intensity pixels of the image, however ignores the entropy or structural information of the image. Moreover, the MSE value is minimum for enhanced image in the proposed technique, indicating more similiarity to the orginal image with minimum loss of information. The clip limit in CLAHE is kept constant at 0.01 , but with the proposed technique the clip limit is chosen adaptively in the range of 0 to 0.01 for each block of size $8 \times 8$, and it is found to present good results. Table 3 shows the performance paramters for the 10 image Datasets.

Table 4: Average values for 50 images

\begin{tabular}{|c|c|c|c|}
\hline Parameter & Fused & CLAHE & CPSO(Proposed) \\
\hline STD & 77.12 & 81.02 & $\mathbf{7 9 . 0 9}$ \\
\hline En & 5.98 & 6.48 & $\mathbf{6 . 2 9}$ \\
\hline PSNR & 22.11 & 20.62 & $\mathbf{2 5 . 7 2}$ \\
\hline SSIM & 0.59 & 0.63 & $\mathbf{0 . 7 8}$ \\
\hline UIQI & 0.59 & 0.57 & $\mathbf{0 . 7 1}$ \\
\hline MSE & -- & 608.51 & $\mathbf{2 3 4 . 6 7}$ \\
\hline CL & -- & 0.01 & $\mathbf{0 . 0}-\mathbf{0 . 0 1}$ \\
\hline
\end{tabular}

\section{5- Conclusion}

A novel technique for enhancement of fused CT and MRI T1 weighted images is proposed. CLAHE is used to enhance the contrast of the fused image and the selection of operational parameters like clip limit, block size and distribution function is automated. The block size is chosen for the experiment as $8 \times 8$. The uniform distribution provides better results for the fused images. The clip limit is in range of $0-0.01$, adaptively chosen by PSO algorithm. The proposed technique improves the visual quality. Further, tumors can be segmented and classified efficiently. Experiments show superior results for PSNR, SSIM, UIQI and MSE. This technique can be applied for enhancement of CT-PET images and MRIPET images. There is an improvement in contrast and entropy for Laplacian Pyramid fused image and CPSO enhanced image by $2.55 \%$ and $5.18 \%$ respectively. CPSO enhanced image indicates an huge increase in PSNR, SSIM and UIQI by $16.32 \%, 32 \%$ and $20.3 \%$ respectively. Also, the MSE has greatly reduced. The proposed technique performs better than CLAHE in terms of PSNR, SSIM, UIQI and MSE.

\section{References}

[1] A.Prof Frank Gaillard et al. "Glioblastoma". Article. https://radiopaedia.org/articles/glioblastoma

[2] Javier E Villanueva, Marc C Mabray, Soonmee Cha, "Current Clinical Brain Tumor Imaging", Neurosurgery 81:397-415, 2017.

[3] Olivier Keunen, Torfinn Taxt, et al., "Multimodal imaging of gliomas in context of evolving cellular and molecular therapies," Adv. Drug Delivery Reviews, Vol.76, 2014, pp. 98-115.

[4] Wolf-Dieter Heiss, Peter Raab, Heinrich Lanferman, "Multimodality Assessment of Brain Tumors and Tumor Recurrence", J Nucl Med 2011, Vol.52, pp.1585-1600.\#\#

[5] Maikel Verduin, Inge Compter, Danny Seijvers et al. "Noninvasive Glioblastoma Testing: Multimodal Approach to Monitoring and Predicting Treatment Response". Hindawi, Disease Markers, Vol. 2018, Article ID 2908609.

[6] Yin Fei, Gao Wei, Son Zongxi. "Medical Image Fusion Based on Feature Extraction and Sparse Representation". Hindawi, International Journal of Biomedical Imaging, Vol. 2017.

[7] Hui Huang, Xi'an Feng, Jionghul Jiang. "Medical Image Fusion Algorithm Based on Nonlinear Approximation of Contourlet Transform and Regional Features". Hindwai Journal of Electrical and Computer Engineering, Vol. 2017.

[8] Hiba Mzoughi, Ines Njeh, et al. "Histogram EqualizationBased Techniques for Contrast Enhancement of MRI Brain Glioma Tumor Images: Comparative Study," in Proc. 4th

[9] International Conference on Advanced Technologies for Signal and Image Processing, ATSIP, March 21-24, 2018.

[10] Bin Yang, Shutao Li, "Multifocus Image Fusion and Restoration with Sparse Restoration," IEEE Transactions on Instrumentation and Measurement, Vol. 59, No.4, 2010.

[11] Robert D Fiete. "Modelling the Imaging Chain of Digital Camera", Image Enhancement Processing, Chap. 9, [Online], SPIE Digital Library.

[12] Yakun Chang, Cheolkon Jung et al. "Automatic ContrastLimited Adaptive Histogram Equalization with Dual Gamma Correction", IEEE Access, Vol. 6, 2010. \#\#

[13] Byong Seok Min, Dong Kyun Lim et al. "A Novel Method of Determining Parameters of CLAHE Based on Image Entropy". International Journal of Software Engineering and Its Applications, Volume 7, No.5, pp. 113120, 2013.

[14] Monika Agarwal and Rashima Mahajan. "Medical Image Contrast Enhancement using Range Limited Weighted Histogram Equalization", in Proc.6th International Conference on Smart Computing and Communication, ICSCC December 2017.

[15] Monika Agarwal and Rashima Mahajan. "Medical Image Contrast Enhancement using Quad Weighted Histogram Equalization with Adaptive Gama Correction and Homomorphic Filtering", in Proc. 7th International Conference on Advances in Computing \& Communications, ICACC-2017.

[16] Youlian Zhu, Cheng Huang. "An Adaptive Histogram Equalization Algorithm on the Image Gray Level Mapping”, in Proc. 2012 International Conference on Solid State Devices and Materials Science, Physics Procedia 25, pp.621-628. 
[17] K Zuiderveld. "Contrast Limited Adaptive Histogram Equalization", in Graphics gems IV, pp474-485, San Diego, CA, USA, Academic Press Professional, Inc. \#\#

[18] Hardeep Kaur, Jyothi Rani. "MRI brain image enhancement using Histogram equalization techniques", in Proc. IEEE WISPNET, 2016.

[19] Kitti Koonsanit, Saowapak Thongvigitmanee, et al. "Image Enhancement on Digital X-Ray Images using NCLAHE", in Proc. IEEE 10th Biomedical Engineering International Conference (BMEiCON), Aug 2017.

[20] Shelda Mohan and T R Mahesh. "Particle Swarm Optimization Based Contrast Limited Enhancement for Mammogram Images", in Proc. 7th International Conference on Intelligent Systems and Control, 2013.

[21] Madhukar Bhat, Tarun Patil M S. "Adaptive Clip Limit for Contrast Limited Adaptive Histogram Equalization (CLAHE) of Medical Images using Least Mean Square Algorithm", in Proc. International Conference on Advance Communication Control and Computing Technologies (ICACCCT), IEEE, 2014.

[22] Luis G More, Macroos A Brizuela et al. "Parameter Tuning of CLAHE-based on Multi-Objective Optimization to Achieve Different Contrast Levels in Medical Images", in Proc. International Conference on Image Processing (ICIP), IEEE 2015.

[23] Sheeba Jennifer, S Parasuraman, Amudha Kadirvelu. "Contrast Enhancement and Brightness preserving of digital mammograms using fuzzy clipped contrast-limited adaptive histogram equalization algorithm", Applied Soft Computing Vol. 42, pp.167-177, May 2016.

[24] Justin Joseph, J Sivaraman, R Periyasamy, V R Simi. “An Objective method to identify optimum clip-limit and histogram specification of contrast limited adaptive histogram equalization for MR Images," Nalecz Institute of Biocybernetics and Biomedical Engineering 37, pp.489497, 2017.

[25] Gurshan Singh, Anand Kumar Mittal. "Controlled Bilateral Filter and CLAHE Based Approach for Image Enhancement," International Journal of Engineering and Computer Science, Volume 3, Issue 11, Nov 2014.

[26] Aziz Makandar, Bhagirathi Halalli. "Breast Cancer Image Enhancement using Median Filter and CLAHE," in Proc. International Journal of Scientific \& Engineering Research, Volume 6, Issue 4, 2015.

[27] Mehemet Zeki, Sarp Erturk. "Enhancement of Ultrasound Images with Bilateral Filter and Rayleigh CLAHE", in
Proc. 23rd Signal Processing and Communications Applications Conference (SIU), Malatya, Turkey, 2015.

[28] James Kennedy and Russell Eberhart. "Particle Swarm Optimization," in Proc. International Conference on Neural Networks, 4, pp.1942-1948, IEEE 1995.

[29] Malik Braik, Alaa Sheta, Aladdin Ayesh. "Image Enhancement Using Particle Swarm Optimization," Proceedings of World Congress on Engineering, Vol. 1, London, UK, 2007.

[30] Leena Chandrashekar, Sreedevi A. "Assessment of NonLinear Filters for MRI Images," in Proc. Second IEEE International Conference on Electrical, Computer and Communication Technologies, Feb 22-24, Coimbatore, India, 2017.

[31] Leena Chandrashekar, Sreedevi A. "A Hybrid Multimodal Medical Image Fusion Technique for CT and MRI brain images," IGI Global International Journal of Computer Vision and Image Processing (IJCVIP), Vol. 8, Issue 3, Sept 2018.

[32] Leena Chandrashekar, Sreedevi A. "A Novel technique for fusing Multimodal and Multiresolution Brain Images," in Proc. 7th International Conference on Advances in Computing and Communications, ICACC 2017, Aug 2224, Cochin, India, 2017.

[33] Leena Chandrashekar, Sreedevi A, "Leena Chandrashekar, A Sreedevi, "A Multi Objective Enhancement Technique for Poor Contrast Magnetic Resonance Images of Brain Glioma blastoma", Third International Conference on Computing and Network Communication, CoCoNet 2019.

Leena Chandrashekar pursing her $\mathrm{PhD}$ in Department of Electrical and Electronics, RVCE, VTU under the guidance of Prof. Sreedevi A. Her research interests include Biomedical Image Fusion, Biomedical image processing, Neural Networks and Deep Learning. She has published papers in international conferences and journals.

Sreedevi A, is working as Associate Professor in Department of Electrical and Electronics Engineering, RVCE, Bangalore, obtained her PhD in the area of Signal Processing. She has published many papers in international conferences and journals. Her research interests include Digital Signal Processing, Digital Image Processing and ControlSystems. 